\begin{tabular}{c} 
International Journal of Engineering \& Technology, $7(2.33)(2018)$ 107-110 \\
International Journal of Engineering \& Technology \\
SPC \\
Website: $w$ ww.sciencepubco.com/index.php/IJET \\
Research paper \\
\hline
\end{tabular}

\title{
Performance analysis of coded 8-pssk system with MRC diversity in fading channels
}

\author{
Jae Young Chun ${ }^{1}$, Kee Soo Yeom ${ }^{2}$, Eon. Gon. Kim ${ }^{2}$ \\ ${ }^{1}$ Graduate School of Information \& Communications. Hanbat National University, Daejeon, Korea \\ ${ }^{2}$ Department of Information \& Communication Eng. Hanbat National University, Daejeon, Korea \\ *Corresponding author E-mail:
}

\begin{abstract}
Due to the increasing demand for low power communication in the field of remote control \& monitoring, it is necessary to introduce a communication technique to meet the various QoS requirements even in an inferior radio channels. In order to ensure these QoS requirements, we propose a communication technique that applies the channel code, diversity scheme into power-efficient modulation. It makes the radio possible to dramatically improve BER by reducing the uncertainty due to fading channels. A core of proposed communication technique is to maximize the performance gain by adopting diversity technique and channel coding scheme. Diversity gain and channel coding gain further increase the performance difference (gain) between power-efficient modulation and conventional modulation, resulting in a signification BER improvement. By adapting the proposed communication technique it is expected to improve the usability of low-power radio according to the satisfaction of the various QoS requirements.
\end{abstract}

Keywords: PSSK Modulation; Convolution Code; MRC Diversity; Rican Fading; Power Efficiency.

\section{Introduction}

Recently, the usability of low power communication due to the convergence of various heterogeneous technologies is gradually increasing (Joonseok Park andMikyeong Moon, 2016). Although a short-range radio communication related to IEEE 802.11 or IEEE 802.15.4 is still highly available, other low-power communication is developed that can be applied in the medium range and long life-cycle. Although not related to global standard such IEEE and ETSI, the dedicated protocols which can be directly connected without link-connection are studied only for these low-power radios. The usability of low-power communication, which is convergence of heterogeneous technologies, is increasing, the type of QoS requirements are gradually diversified. In order to meet these QoS demands, the performance improvement of low-power radio is basically required.

Most of low-power radio devices transmit the data stream at transmit power of $10 \mathrm{~mW}$ (Jose A. Gutierrez and Edgar H. Callaway, 2004). Since the electromagnetic waves are used, the replica signals reach the receiving station via multiple paths. The problem is that these replica signals cause serious signal distortion when synthesized at the receiving station. Assuming that the replica signals pass through independent paths, it is possible to reduce the fading influence by using diversity scheme primarily. Next, by applying the channel coding scheme, hidden errors can be corrected; therefore, it is possible to achieve an additional performance gain. The proposed communication technique applied the diversity technique and the channel coding scheme into power-efficient modulation to maximize the performance gain.

In this paper, PSSK modulation is adopted as a basic modulation platform. As PSSK is a kind of hybrid modulation technique combining PPM and PSK(D. K. Kim and H. S. Lee, 2009). It includes a silence period in a symbol period and applies a lower one-step modulation order, allowing it to perform better than a PSK with the same modulation order (Edward A. Lee and David G. Messerschmitt, 1993). MRC diversity is adopted to aggressively suppress the fading influence. MRC diversity provides a method to maximize a performance gain by intentionally weighting and synthesizing the received signals of all branches (Murat Torlak, 2017). MRC diversity has an advantage of producing output with an acceptable SNR even when none of individual signals are themselves acceptable (John G. Proakis, 1997). Convolution codes is adopted to achieve an additional performance gain. A Convolution code provides a method to identify and correct hidden errors the mathematically most likely message from among the set of all possible transmitted messages (BERNARD SKLAR. 2001). Therefore, it is possible to avoid deep fading and achieve performance gain simultaneously. As a result, the performance difference between PSSK and PSK system become larger due to diversity and channel code effects. In order to verify the performance enhancements of the proposed communication technique, we evaluate the BER curves through computer simulation and derive the performance equations. 


\section{Communication model and performance analysis}

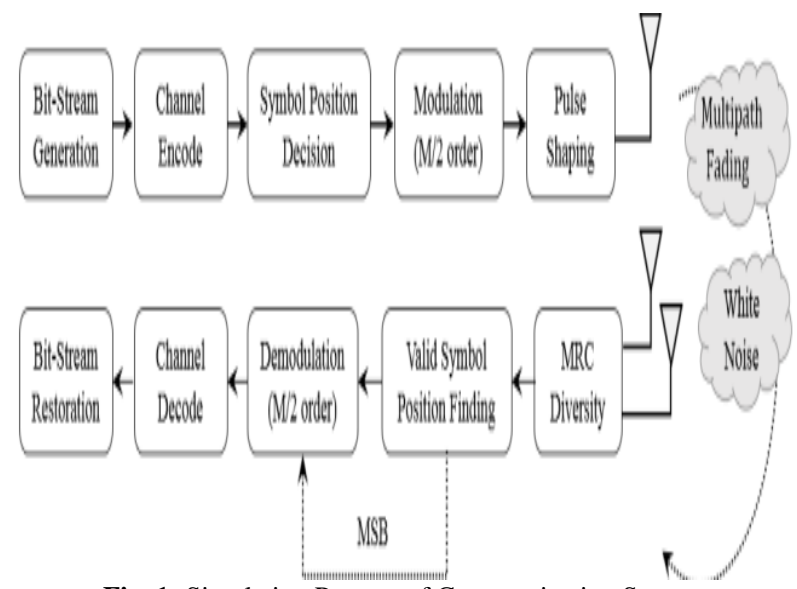

Fig. 1: Simulation Process of Communication System.

Fig. 1 illustrates the simulation process of PSSK system using schematic diagram. First, binary data are input to convolution encoder in transmit stage. Here, coding rate is $1 / 2$, constrain length is $\mathrm{K}=$ [7], and the generators are [171], [133] (Begin, G.et al., 1990). PSSK is one of the derivative modulation scheme combining PSK and PPM. PSSK modulator determines the symbol position in symbol period according to MSB value. Then, the modulated signals are transmitted after pulse shaping with SRRC filter. The transmitted signals are corrupted by multipath fading and white noise. The fading model assumes Rician channels. In this paper, it is assumed that each of signals is received via an independent path. MRC diversity can capture and synthesize these signal via independent paths using multiple antenna. Here, MRC is a diversity scheme that maximized the SNR by multiplying all signals by a weight coefficient. Then, the post-processed signals at diversity stage is delivered to the demodulator. Finally, Viterbi decoder is used to decode the bit-stream encoded with the convolution code.

M-ray PSSK modulation is the combination of M/2-ary PSK and PPM. M-ary PSSK firstly examines the most significant bits of binary data, and then PSSK modulator maps M/2-ary PSK symbols into the valid symbol position (D. K. Kim and H. S. Lee, 2009).
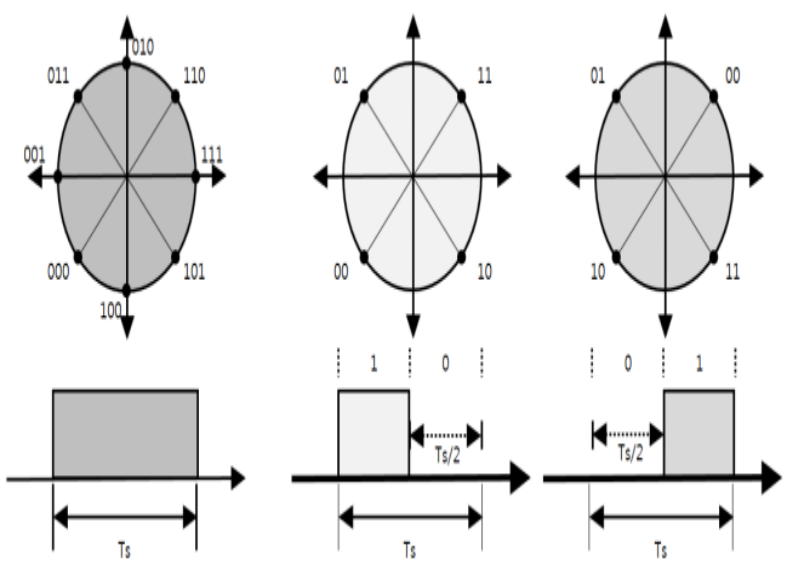

Fig. 2: Constellation and Symbol Duration for (A) 8-PSK and (B) 8 PSSK.

In Fig. 2(b), PSSK symbol duration is T/2 while PSK symbol period is T as Fig. 2(b). Symbol error probabilityof 8-PSSK signal in AWGN is derived as follows (Jung-Yeol Oh, 2010).

$$
\mathrm{P}(\mathrm{e})=6 \mathrm{Q}(3 \gamma)
$$

Where $\gamma$ is bit energy per noise power spectral density ratio $E_{b} / N_{0}$.
In the considered communication systems, the wireless channel is modeled as frequency non-selective slow Rician fading corrupted by the AWGN process. The fading processes in the MRC diversity channels are assumed to be mutually independent. The noise processes are also assumed to be independent, with identical autocorrelation functions. In Rician fading, the output instantaneous CNR of MRC diversity becomes a random variable and its PDF can be calculated as follows (I. S. Gradshteyn and I. M. Rhyzhik, 1994).

$\mathrm{P}_{\mathrm{MRC}}\left(\gamma_{\mathrm{S}}\right)=\left(\frac{\mathrm{K}+1}{\Gamma_{\mathrm{i}}}\right)^{\frac{\mathrm{L}+1}{2}}\left(\frac{\gamma_{\mathrm{S}}}{\mathrm{K} \cdot \mathrm{L}}\right)^{\frac{\mathrm{L}-1}{2}}$

$\times \mathrm{e}^{\left(-\mathrm{K} \cdot \mathrm{L}-\frac{\mathrm{K}+1}{\Gamma_{\mathrm{i}}} \gamma_{\mathrm{s}}\right)} \mathrm{I}_{\mathrm{L}-1}\left(2 \sqrt{\frac{\mathrm{K}(\mathrm{K}+1) \mathrm{L} \cdot \gamma_{\mathrm{s}}}{\Gamma_{\mathrm{i}}}}\right)$

Where $\Gamma_{i}$ denotes the input average CNR. K represents the Rician parameter. Further, $\mathrm{I}_{(\mathrm{L}-1)}$ refers to the modified Bessel function of the first kind and order (L-1).

The output average CNR of MRC diversity, $\Gamma_{\mathrm{o}}$ can be derived as follows:

$\Gamma_{\mathrm{o}}=\int_{0}^{\infty} \gamma_{\mathrm{s}} \mathrm{P}_{\mathrm{MRC}}\left(\gamma_{\mathrm{s}}\right) \mathrm{d} \gamma_{\mathrm{s}}=\mathrm{L} \cdot \Gamma_{\mathrm{i}}$

Assuming that the received 8-PSSK signal corrupted by Rician fading in each diversity branch is uncorrelated, we derive the SER of 8-PSSK with MRC diversity reception by considering the variations of the instantaneous CNR and average CNR between the input and the output of the MRC diversity circuit as follows:

$$
\left.\mathrm{P}_{\mathrm{MRC}}=3\left\{\begin{array}{c}
1-\frac{2}{\sqrt{\pi}} \sum_{\mathrm{K}=0}^{\infty} \frac{(-1)^{\mathrm{K}}}{\mathrm{K} !(2 \mathrm{~K}+1) 2^{\mathrm{K}+\frac{1}{2}}} \times \\
\sum_{\mathrm{m}=0}^{\infty} \frac{1}{\mathrm{~m} ! \cdot \Gamma(\mathrm{m}+\mathrm{L})}\left(\frac{\mathrm{K}+1}{\Gamma_{\mathrm{i}}}\right)^{\mathrm{m}+\mathrm{L}} \\
\times(\mathrm{KL})^{\mathrm{m}} \mathrm{e}^{-\mathrm{KL}}\left(\frac{\mathrm{K}+1}{\Gamma_{\mathrm{i}}}\right)^{\mathrm{K}+\mathrm{L}+\mathrm{m}+\frac{1}{2}} \\
\times \Gamma\left(\mathrm{K}+\mathrm{L}+\mathrm{m}+\frac{1}{2}\right)
\end{array}\right\}\right\}
$$

Where $\Gamma(\cdot)$ is Gamma function.

The performance of coded 8-PSSK system with MRC diversity is simulated using computer simulation with matlab in Rician fading channel.

\section{Results and discussion}

In this section, some simulation results for the proposed communication technique are presented using BER curve. As shown in the following table, Convolution Code and MRC diversity are used as basic communication technique in fading channels. To verify the superior performance of the proposed communication technique, we confirm BER of coded PSSK system with MRC diversity by Rician $\mathrm{K}$ factor.

Table 1: Parameters of 8-Pssk System

\begin{tabular}{ll}
\hline Parameter & Value \\
\hline Modulation Type & PSK and PPM \\
Modulation Order & 8 \\
Symbol Rate & $3.0 \mathrm{Msps}$ \\
Diversity Type & 2 -ary MRC \\
Channel Coding Type & Convolution Code $(7,[171,133])$ \\
Fading Channel & Rician Channels $(\mathrm{K}=0 \sim 9)$ \\
\hline
\end{tabular}

Fig. 3 presents the BER of coded 8-PSSK system with 2-ary MRC diversity in Rician channels. Fading depth can be set by steering the Rician $\mathrm{K}$ factor. Here, Code rate is $1 / 2$, constraint length is 7 and code generator is [171], [133]. As Rician K factor increases, the fading depth also tends to decrease. As shown this figure, the BER curves of PSSK system is clearly distinguished from BER curves of PSK system according to $\mathrm{E}_{\mathrm{b}} / \mathrm{N}_{0}$. Also as the Rician K- 
factor increases, the BER performance of both PSK and PSSK systems improved overall.

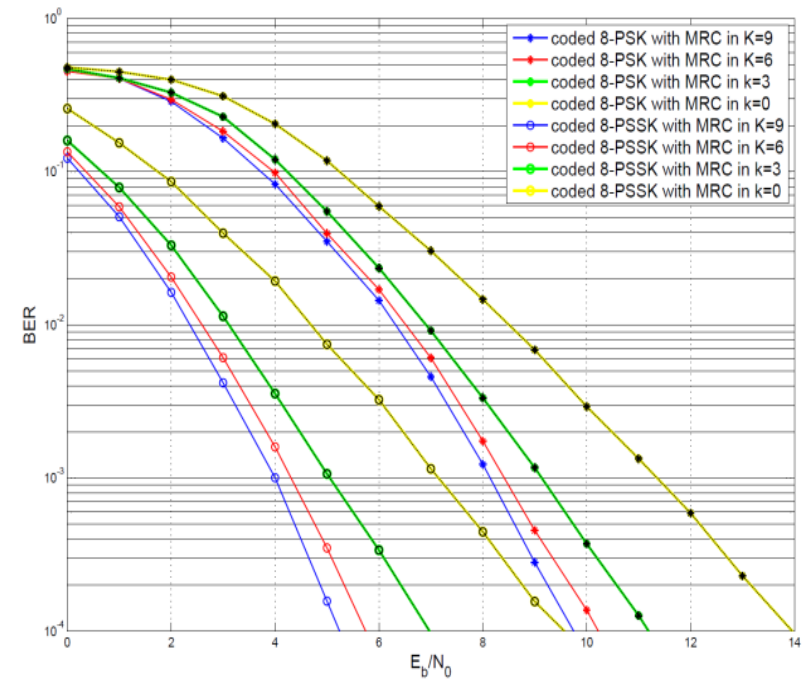

Fig. 3: BER of 8-PSK System and 8-PSSK System in Rician Fading Channels.

Fig. 4 presents the BER curves of and 8-PSSK system with or without MRC diversity or Convolution Coding in Rayleigh fading channel. Also, to demonstrate the performance enhancement due to the PSSK system, a comparative simulation with 8-PSK system having the same condition as 8-PSSK system is performed. It can be seen that the performance improvement is very high when applied together rather than separately applying the MRC diversity and convolution coding. $\mathrm{E}_{\mathrm{b}} / \mathrm{N}_{0}$ is $14.9 \mathrm{~dB}$ in $\mathrm{BER}=10^{-3}$ when only channel coding is applied, and $\mathrm{E}_{\mathrm{b}} / \mathrm{N}_{0}$ is $15.1 \mathrm{~dB}$ in $\mathrm{BER}=10^{-3}$ when only diversity is applied. And $\mathrm{E}_{\mathrm{b}} / \mathrm{N}_{0}$ is $7.1 \mathrm{~dB}$ in BER when channel coding and diversity are applied. Even though Rayleigh fading which is deep fading, it can be seen that hybrid effects of convolutional coding and MRC diversity is very significant.

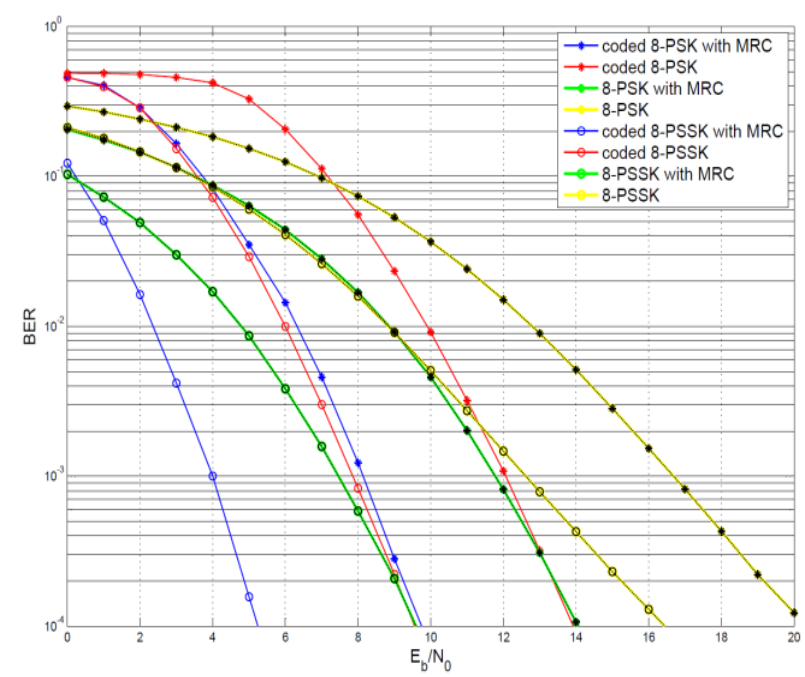

Fig. 4: BER Comparison of 8-PSK System and 8-PSSK System in Rayleigh Fading Channel.

Fig. 5 presents the BER curves of and 8-PSSK system with or without MRC diversity or Convolution Coding in Rician fading channel $(\mathrm{K}=9)$. This simulation is the same as the preceding Fig. 4, except that the fading channel is different. As expected, synergy effect related to the BER performance are significant when both convolution coding and diversity techniques are applied. The 8PSSK system without channel coding and diversity technique has as $\mathrm{BER}=10^{-3}$ at

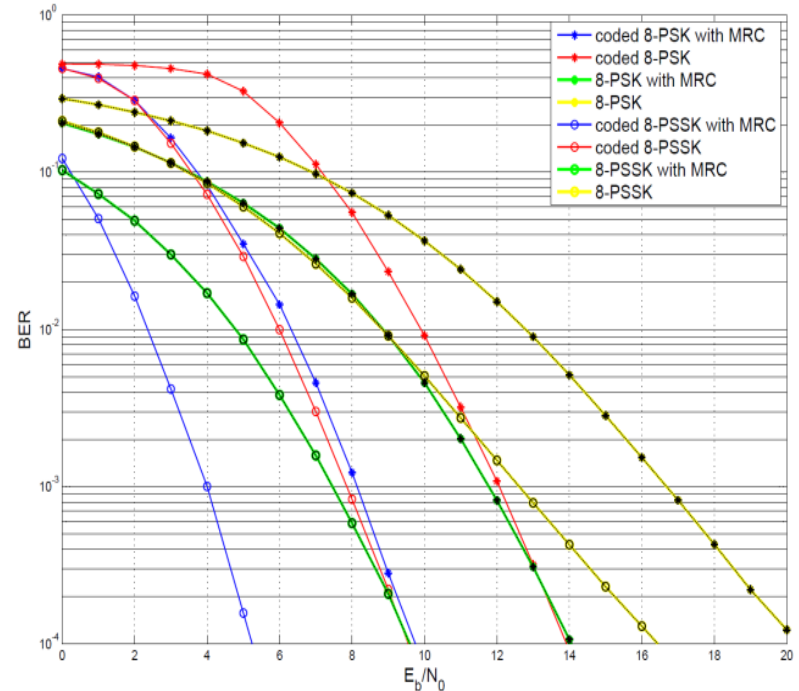

Fig. 5: BER Comparison of 8-PSK System and 8-PSSK System in Rician Fading Channel $(\mathrm{K}=9)$.

$\mathrm{E}_{\mathrm{b}} / \mathrm{N}_{0}=12.6 \mathrm{~dB}$, while the PSSK system with both channel coding and diversity technique shows a significant performance improvement at $\mathrm{BER}=10^{-3}$ at $\mathrm{E}_{\mathrm{b}} / \mathrm{N}_{0}=4.0 \mathrm{~dB}$.In case of the8-PSK system, the 8-PSK system without channel coding and diversity technique has as $\mathrm{BER}=10^{-3}$ at $\mathrm{E}_{\mathrm{b}} / \mathrm{N}_{0}=16.6 \mathrm{~dB}$, while the 8-PSK system with both channel coding and diversity technique shows similarly significant performance improvement at $\mathrm{BER}=10^{-3}$ at $\mathrm{Eb} / \mathrm{N}_{0}=8.1 \mathrm{~dB}$

In summary, although the channel coding and the diversity technique can achieve the performance improvement only by themselves, it can be seem from Fig. 4 to 5 , that the performance improvement is great when both of them are applied. In addition, we confirmed that the fading influence is reduced when channel coding and diversity scheme is applied to this 8-PSSK system.

\section{Conclusion}

A In this paper, we propose a communication technique which applies MRC diversity and Convolution Coding to 8-PSSK modulation. By adopting 8-PSSK instead of 8-PSK, a performance gain of $3 \sim 4 \mathrm{~dB}$ can be achieved. Here, if diversity gain and channel coding gain are applied together, the performance difference between 8-PSSK system and 8-PSK becomes larger. The simulation results are summarized in Fig. 3 to 5, and it can be confirmed that there is a significant performance difference depending on whether or not the channel coding and the diversity technique are applied. In summary, we have confirmed through computer simulation that the proposed communication technique can contribute to improving the QoS of low power communication.

\section{References}

[1] Begin, G., Haccoun, D., and Paquin, Nov. 1990, Further results on High-Rate Punctured Convolutional Codes for Viterbi and Sequential Decoding, IEEE Transactions on Communications, Vol. 38, No. 11, p. 1923.

[2] BERNARD SKLAR. 2001. Digital Communications Fundamentals and Applications 2nd, Prentice Hall, pp. 978 988.

[3] D. K. Kim, H. S. Lee, Jun. 2009. Phase-silence-shift-keying for power-efficient modulator, IEICE Trans. Com, vol. E92-B, no. 6 , pp. 2324-2326

[4] Edward A. Lee, David G. Messerschmitt, Aug. 1993. Digital Communication: Second Edition, Springer, pp. 148-160, 213-220.

[5] I. S. Gradshteyn and I. M. Rhyzhik, 1994. Table of Integrals, Series and Products, San Diego, cA: Academic Press, 5th Ed.

[6] John G. Proakis, 1997. Digital Communication: third edition, McGraw-Hill, Inc., pp. 165-173, 282- 284.

[7] Joonseok Park, Mikyeong Moon. June 2016. Method for Ensuring Stable IoT Services based on SDN, Indian Journal of Science and Technology,Vol9(24), DOI:10.17485/ijst/2016/v9i24/96155. 
[8] Jose A. Gutierrez,Edgar H. Callaway,2004. Jr. Low-Rate Wireless Personal Area Networks: Enabling Wireless Sensors with IEEE 802.15.4, IEEE published by Standard Information Network. ISBN 0-7381-3557-7.

[9] Jung-Yeol Oh, MAY 2010. PSSK Modulation Scheme for HighData Rate Implantable Medical Devices, IEEE Transactions on Information Technology in Biomedicine, VOL. 14, NO. 3.

[10] Prof. Murat Torlak, Diversity Techniques (EE4365), (http://www.utdallas.edu/ saquib/EE4365/Diversity/diversityEE4365.pdf). 